\title{
Using Difference Scheme Method to the Fractional Telegraph Model with Atangana-Baleanu-Caputo Derivative
}

Mahmut Modanli

Harran University, Faculty of Arts and Sciences, Department of Mathematics, Sanliurfa, Turkey

mmodanli@harran.edu.tr,

\begin{abstract}
The fractional telegraph partial differential equation with fractional Atangana-Baleanu-Caputo (ABC) derivative is studied. Laplace method is used to find the exact solution of this equation. Stability inequalities are proved for the exact solution. Difference schemes for the implicit finite method are constructed. The implicit finite method is used to deal with modelling the fractional telegraph differential equation defined by Caputo fractional of Atangana-Baleanu (AB) derivative for different interval. Stability of difference schemes for this problem is proved by the matrix method. Numerical results with respect to the exact solution confirm the accuracy and effectiveness of the proposed method.
\end{abstract}

Keywords: Fractional Telegraph Model with Atangana-Baleanu derivative; Laplace method; Stability inequalites; Difference schemes; Implicit finite method

This research deal with the application of Laplace method to the Fractional Telegraph partial differential equation defined by fractional Atangana-Baleanu-Caputo (ABC) derivative. Moreover, Difference schemes for Implicit method are constructed. The stability of difference schemes for this problem is newly presented by matrix method. The numerical simulation with respect to $\mathrm{ABC}$ derivative is performed. These results with respect to the exact solution confirm the accuracy and effectiveness of the proposed method. Furthermore, the surfaces and numerical results newly obtained in this paper show that the expected physical properties of the model are also observed.

\section{Introduction}

Over the last several decades, many scientists and experts are turning their studies into real world problems. This trend comes from new and novel structures of models to the real world problems and the application of differential equations with fractional derivative $[1,2,3,4]$. Therefore, many fractional theorems and definitions in the sense of Rimann-Liouville, Caputo, Atangana-Baleanu have been introduced to the literature. Moreover, a variety of many interesting tools by modifiying and developing such as $\left(\mathrm{G}^{\prime} / \mathrm{G}^{\wedge}\{2\}\right)$-expansion method, homotopy analysis and perturbation methods, Fractional Adams-Bashforth-Moulton 
method, Reduced differential transform method, variational iteration method, Adomian's decomposition method, Exp-function method, (G'/G)-expansion method, finite forward difference method, modified simple equation method and so on $[5,6,7]$ have been presented to find the analytical solutions of fractional differential equations. Common properties of these methods preserve generally the nonlinearities along with definitions and theorems in the mathematical and physical points of view.

In this sense, fractional differential equation has several applications in engineering, finance, physics and seismology [8, 9, 10]. This differential equation is solvable with respect to variables time and space. In [11], the telegraph differential equation was studied for $\alpha=1$ and $0<x<\pi$ by using difference scheme method. In [12, 13], Modanli studied two different numerical methods for the fractional telegraph differential equation. The AB derivative is applied successfully in modelling of various real phenomena such as $[14,15,16]$. Atangana and Baleanu suggested another version of fractional-order derivative which uses the generalized Mittag-Leffler function as non-local and non-singular kernel in [17]. Some important papers published depend on fractional order differential equation in recent years $[18,19]$. Finally, the important works were presented involving the recent advancements in fractional calculus and its applications $[20,21]$.

The implicit finite difference method has signficant advantages. One of these advantages is that stability can be ensured over much larger values of $\Delta t$ and fewer time steps are needed to carry out the calculations over a given interval [22]. Sometimes differential equations are very difficult to solve analytically or models are needed for computer simulations. In these cases finite-difference methods are used to solve the equations instead of analytical ones. Finitedifference methods are numerical methods for solving differential equations by approximating them with difference equations, in which finite differences approximate the derivatives. For example, in electronics and electrical engineering, differential equations describing complex circuits containing capacitors, inductors and resistors can be replaced with finite-difference equations. Computer simulations of the models are used to estimate voltages and currents in the nodes of the circuits.

Perturbation methods are mostly based on small (or large) physical parameters, called perturbation quantity. Using small or large physical parameters, perturbation methods transfer a nonlinear equation into an infinite number of subproblems that are mostly linear. Unfortunately, many nonlinear equations do not contain such kind of perturbation quantities at all. More importantly, perturbation approximations often quickly become invalid when the so-called perturbation quantities enlarge. Besides to these facts, perturbation techniques are so strongly dependent upon physical small parameters that we have nearly no freedom to choose equation type and solution expression of high-order approximation equations, which are often complicated and thus difficult to solve. Due to these restrictions, perturbation methods are valid mostly for weakly nonlinear problems in general. In theory, it is very valuable to develop a new kind of analytic approximation method which should have the following characteristics: 
(1) it is independent of small physical parameter;

(2) it provides us great freedom and flexibility to choose the equation-type and solution expression of high-order approximation equations;

(3) it provides us a convenient way to guarantee the convergence of approximation series [23, 24].

In this article, we consider fractional telegraph differential equation model with $\mathrm{AB}$ derivative. The implicit method is used for the fractional telegraph pde by defined $\mathrm{AB}$ derivative to obtain numerical results. Then, the matrix stability method is showed by the given conditions. We shall examine the following fractional telegraph equation

$$
\left\{\begin{array}{l}
\frac{\partial^{2} u(t, x)}{\partial t^{2}}+{ }_{0}^{A B C} D_{t}^{\alpha} u(t, x)-\frac{\partial^{2} u(t, x)}{\partial x^{2}}=f(t, x) \\
0<x<L, 0<t<T \\
u(0, x)=h_{1}(x), u_{t}(0, x)=h_{2}, 0 \leq t \leq T \\
u\left(t, X_{L}\right)=u\left(t, X_{R}\right)=0, X_{L}<x<X_{R} \\
0<\alpha \leq 1
\end{array}\right.
$$

Now, we shall recall some basic definitions and properties of fractional calculus theory for fractional telegraph partial differential equation. Difference schemes of the implicit finite difference method is defined for this differential equation.

Definition 1 The Caputo fractional derivative $D_{t}^{\alpha} u(t, x)$ of order $\alpha$ with respect to time is defined as:

$$
\begin{aligned}
\frac{\partial^{\alpha} u(t, x)}{\partial t^{\alpha}} & =D_{t}^{\alpha} u(t, x) \\
& =\frac{1}{\Gamma(n-\alpha)} \int_{0}^{t} \frac{1}{(t-p)^{\alpha-n+1}} \frac{\partial^{\alpha} u(p, x)}{\partial p^{\alpha}} d p, \quad(n-1<\alpha<n),
\end{aligned}
$$

and for $\alpha=n \in N$ defined as:

$$
D_{t}^{\alpha} u(t, x)=\frac{\partial^{\alpha} u(t, x)}{\partial t^{\alpha}}=\frac{\partial^{n} u(t, x)}{\partial t^{n}} .
$$

Definition 2 Let $f \in H^{1}(a, b), b>a, \alpha \in[0,1]$ then, the definition of the new fractional derivative (Atangana-Baleanu derivative in Caputo sense) is ginev as:

$$
{ }_{a}^{A B C} D_{t}^{\alpha}(u(t))=\frac{B(\alpha)}{1-\alpha} \int_{0}^{t} f^{\prime}(x) E_{\alpha}\left[-\alpha \frac{(t-x)^{\alpha}}{1-\alpha}\right] d x
$$


where $B(\alpha)=1-\alpha+\frac{1}{\Gamma(\alpha)}$, and

$$
E_{\alpha}\left[-\alpha \frac{(t-x)^{\alpha}}{1-\alpha}\right]=\sum_{k=0}^{\infty} \frac{\left(-\alpha \frac{(t-x)^{\alpha}}{1-\alpha}\right)^{k}}{\Gamma(\alpha k+1)} .
$$

Atangana-Baleanu computate the Laplace transform for the equation (4) the following form as:

$$
£\left\{{ }_{a}^{A B C} D_{t}^{\alpha}(u(t))\right\}=\frac{B(\alpha)}{1-\alpha} \frac{U(s) s^{\alpha}-s^{\alpha-1} u(0)}{s^{\alpha}+\frac{\alpha}{1-\alpha}} .
$$

In the present paper, the exact solution of the fractional order telegraph partial differential equation for abstract form is obtained and for this exact solution is presented the stability estimates. The first order of accuracy implicit finite difference schemes are presented for the approximate solution of problem (1). The stability estimates for the solution of these implicit finite difference schemes are obtained. A numerical method is proposed for solving initial-boundary value problem for the fractional order telegraph partial differential equation. A procedure of modified Gauss elimination method is used for solving these difference schemes in the case of the fractional order telegraph partial differential equations.

The detail of the remaining sections of this paper is as follows: In section 1, the basic definitions of $\mathrm{AB}$ derivative is presented. The fractional telegraph model formulation by applying the fractional calculus is presented and stability inequalities are showed for fractional telegraph model with $\mathrm{AB}$ derivative in section 2. In section 3, Difference schemes are constructed for implicit difference scheme method and stability estiates are proved for given method. Numerical results are given by Implicit method in section. 4. Finally, the present work is concluded in section 5 .

\section{Exact solution of fractional telegraph differ- ential equation and its stability estimates}

In this section, we find the exact solution for the abstract form of the equation (1). Then, we prove the stability estimates for the equation (6).

Problem (1) can be rewritten in the following form:

$$
\left\{\begin{array}{l}
\frac{d^{2} u(t)}{d t^{2}}+{ }_{a}^{A B C} D_{t}^{\alpha}(u(t))+K u(t)=g(t),(0<t<T), \\
u(0)=h_{1}, u^{\prime}(0)=h_{2}, 0 \leq \alpha \leq 1
\end{array}\right.
$$

in a Hilbert space $H=L_{2}[0, L]$. Here, $f(t)=f(t, x)$ is given abstract function defined on $[0, T]$ with values in $H=L_{2}[0, L] . h_{1}=h_{1}(x)$ and $h_{2}=h_{2}(x)$ are the elements of $H=L_{2}[0, L] . u(t)=u(t, x)$ is the unknown abstract function 
defined on $[0, T]$ with values in $H=L_{2}[0, L] . K: D(K) \rightarrow H$ is the differential operator defined by

$$
K u(x)=-u^{\prime \prime}(x)
$$

with domain

$$
D(K)=\left\{u: u, u^{\prime}, u^{\prime \prime} \in L_{2}[0, L] ; u(0)=u(L)=0\right\} .
$$

Here, we have that $\langle K u, v\rangle=\langle u, K v\rangle(u, v \in D(K))$ and $\langle K u, u\rangle \geq \delta\langle u, u\rangle$, $\delta>0, \delta \geq 0$.

Now, we shall obtain the exact solution of the problem (6). Taking $G(t)=$ $g(t)-{ }_{a}^{A B C} D_{t}^{\alpha}(u(t))$, the formula (6) is rewritten as follows:

$$
\left\{\begin{array}{l}
\frac{d^{2} u(t)}{d t^{2}}+K u(t)=G(t), \quad(0<t<T) \\
u(0)=h_{1}, u^{\prime}(0)=h_{2}, 0 \leq \alpha \leq 1
\end{array}\right.
$$

A function $u(t)$ is called a solution of the problem (7) if the following conditions are satisfied:

(i) $u(t)$ is a twice continuously differentiable on the interval $[0, T]$. The derivatives at the endpoints of the interval are understood as the appropriate unilateral derivatives.

(ii) The value $u(t)$ relates to $D(K)$ for all $t \in[0, T]$ and the function $K u(t)$ is continuous on the interval $[0, T]$.

(iii) $u(t)$ satisfies the initial conditions (7).

Then, for the exact solution we can rewrite the problem (7) as the following the first order differential equations systems forms

$$
\left\{\begin{array}{c}
\frac{d u(t)}{d t}+i L u(t)=y(t) \\
\frac{d y(t)}{d t}-i L z(t)=G(t) \\
y(0)=u^{\prime}(0)+i L u(0)
\end{array}\right.
$$

where, $L=K^{1 / 2}$. Here, $y(t)$ is a continuously differentiable on the interval $[0, T]$. Integrating the formula $(8)$, we get

$$
\left\{\begin{array}{l}
u(t)=e^{-i K t} u(0)+\int_{0}^{t} e^{-i K(t-y)} y(s) d s \\
y(t)=e^{i L t} y(0)+\int_{0}^{t} e^{i L(t-y)} G(s) d s
\end{array}\right.
$$

Applying the initial condition $y(0)=u^{\prime}(0)+i K^{\frac{1}{2}} u(0)$, we can get

$$
u(t)=e^{-i L t} u(0)+\int_{0}^{t} e^{-i L(t-s)} \int_{0}^{s} e^{i L(y-p)} G(p) d p d s
$$




$$
\begin{aligned}
& +\int_{0}^{t} e^{-i L(t-s)} e^{i L s} d s\left(u^{\prime}(0)+i L u(0)\right) \\
= & e^{-i L t} u(0)+\left(i L \int_{0}^{t} e^{-i L(t-y)} e^{i L s} d s\right) u(0)+\int_{0}^{t}\left(e^{-i L(t-y)}-e^{i L s}\right) d s u^{\prime}(0) \\
& +\int_{0}^{t} \int_{0}^{s} e^{-i L(t-y)} e^{i L(s-p)} G(p) d p d s .
\end{aligned}
$$

By an interchange of the order integration, we can write

$$
\begin{aligned}
u(t)= & \left(e^{-i L t}+\frac{e^{i L t}-e^{-i L t}}{2}\right) u(0)+\left(B \frac{e^{i L t}-e^{-i L t}}{2 i}\right) u^{\prime}(0) \\
& +\int_{0}^{t} \int_{p}^{t}\left(e^{-i L(t-y)} e^{i L(s-p)}\right) G(p) d s d p \\
= & \left(e^{-i L t}+\frac{e^{i L t}-e^{-i L t}}{2}\right) u(0)+\left(L^{-1} \frac{e^{i L t}-e^{-i L t}}{2 i}\right) u^{\prime}(0) \\
& +\int_{0}^{t} L^{-1} \frac{e^{i L(t-y)}-e^{-i L(t-y)}}{2 i} G(s) d s .
\end{aligned}
$$

Then, we obtain

$$
u(t)=c(t) u(0)+s(t) u^{\prime}(0)+\int_{0}^{t} s(t-s) G(s) d s
$$

where

$$
L=K^{\frac{1}{2}}, c(t)=\frac{e^{i K^{\frac{1}{2}} t}+e^{-i K^{\frac{1}{2}} t}}{2}, s(t)=K^{-\frac{1}{2}} \frac{e^{i K^{\frac{1}{2}} t}-e^{-i K^{\frac{1}{2}} t}}{2 i} .
$$

Using the formula (9) and the formula $G(t)=g(t)-{ }_{0}^{A B C} D_{t}^{\alpha}(u(t))$, we obtain

$$
u(t)=c(t) u(0)+s(t) u^{\prime}(0)+\int_{0}^{t} s(t-y) g(y) d y-\int_{0}^{t} s(t-y)_{0}^{A B C} D_{t}^{\alpha}(u(y)) d y .
$$

Using the formula (3), we can write 


$$
\begin{aligned}
\left\|_{0}^{A B C} D_{t}^{\alpha}(u(t))\right\| & =\frac{B(\alpha)}{1-\alpha}\left\|\int_{0}^{t} u^{\prime}(p) E_{\alpha}\left[-\alpha \frac{(t-p)^{\alpha}}{1-\alpha}\right] d p\right\| \\
& =\frac{B(\alpha)}{1-\alpha}\left\|\int_{0}^{t} u^{\prime}(p) E_{\alpha}\left[-\alpha \frac{(t-p)^{\alpha}}{1-\alpha}\right] d p\right\| .
\end{aligned}
$$

Using the partial integration for the last formula (cf. [25] ) and properties Mittag-Leffler function, we get

$$
\begin{aligned}
\left\|{ }_{0}^{A B C} D_{t}^{\alpha}(u(t))\right\|= & \frac{B(\alpha)}{1-\alpha} \|-u(t) E_{\alpha}\left[-\alpha \frac{t^{\alpha}}{1-\alpha}\right] \\
& +\sum_{k=0}^{\infty} \frac{\left(-\frac{\alpha}{1-\alpha}\right)^{k}}{\Gamma(\alpha k+1)} \alpha k \int_{0}^{t} u(p)(t-p)^{\alpha k-1} d p \| \\
\leq & M\|u(t)\| .
\end{aligned}
$$

Thus, we can give the following theorem for the stability estimates the equation (11).

Theorem 3 Let $h_{1} \in D(K), h_{2} \in D\left(K^{1 / 2}\right)$ and $g(t)$ be continuously differentiable on $[0, T]$. Then, there exists a unique solution of problem (11) and the following stability estimate is satisfied:

$$
\begin{gathered}
\max _{0 \leq t \leq T}\|u(t)\|_{H} \\
\left.\leq M_{1}(\alpha, \delta)\left\{\left\|h_{1}\right\|_{H}+\left\|K^{-1 / 2} h_{2}\right\|\right)_{H}+\max _{0 \leq t \leq T}\left\|K^{-1 / 2} g(t)\right\|_{H}\right\} .
\end{gathered}
$$

Proof. Using the formulas (11), (10) and the estimates (12), we obtain the following estimates

$$
\begin{aligned}
\|u(t)\|_{H} \leq & \|c(t)\|_{H \rightarrow H}\|u(0)\|_{H}+\left\|K^{-1 / 2} K^{1 / 2} s(t)\right\|_{H}\left\|K^{-1 / 2} u^{\prime}(0)\right\|_{H} \\
& +\int_{0}^{t}\left\|K^{1 / 2} s(t-y)\right\|_{H \rightarrow H}\left\|K^{-1 / 2} g(y)\right\|_{H} d y \\
& +\int_{0}^{t}\left\|K^{1 / 2} s(t-y)\right\|_{H \rightarrow H}\left\|K_{0}^{-1 / 2 A B C} D_{t}^{\alpha}(u(y)) d y\right\|_{H} d y \\
\leq\|u(0)\|_{H} & +\left\|K^{-1 / 2} u^{\prime}(0)\right\|_{H}+\max _{0 \leq t \leq T}\left\|K^{-1 / 2} g(y)\right\|_{H}+\delta^{-\frac{1}{2}} M\|u(t)\|_{H} .
\end{aligned}
$$


From the initial condition, we can obtain the estimate as the formula (13). Thus, the proof of theorem is completed.

The last part of this section, we give a test example of the fractional order telegraph differential equation for the exact solution by the Laplace transform method.

Example 4 Consider the following fractional order telegraph differential equation with defined by $A B$ derivative

$$
\left\{\begin{array}{l}
\frac{\partial^{2} u(t, x)}{\partial t^{2}}+{ }_{0}^{A B C} D_{t}^{\alpha} u(t, x)-\frac{\partial^{2} u(t, x)}{\partial x^{2}}=f(t, x) \\
f(t, x)=\left(\frac{2(1-\alpha)}{B(\alpha)}\left(1+t^{2}\right)+\frac{2}{\Gamma(\alpha) B(\alpha)} t^{\alpha}+t^{2}+\frac{2 \alpha}{\Gamma(\alpha+3) B(\alpha)} t^{\alpha+2}\right) \sin x \\
0<x<\pi, 0<t<1 \\
u(0, x)=u_{t}(0, x)=0,0 \leq t \leq 1 \\
u(t, 0)=u(t, \pi)=0,0 \leq x \leq \pi \\
0 \leq \alpha \leq 1
\end{array}\right.
$$

Solution 5 Applying the Laplace transform method both to sides the equation (14) and using the given initial condition, we obtain

$$
\begin{aligned}
& \left(s^{2}+\frac{B(\alpha)}{1-\alpha} \frac{s^{\alpha}}{s^{\alpha}+\frac{\alpha}{1-\alpha}}\right) u(s, x)-\frac{\partial^{2} u(s, x)}{\partial x^{2}} \\
= & \frac{2}{s^{3}}\left(\frac{s^{\alpha}(1-\alpha+B(\alpha))+\alpha+s^{2}\left(s^{\alpha}(1-\alpha)+\alpha\right)}{s^{\alpha} B(\alpha)} \sin x .\right.
\end{aligned}
$$

Solving this equation for homogenous and nonhomogenous part as to $x$, and using boundary value conditions, we have

$$
u(s, x)=\frac{2}{s^{3}}\left(\frac{1-\alpha}{B(\alpha)}+\frac{\alpha}{s^{\alpha} B(\alpha)}\right) \sin x .
$$

Taking the inverse Laplace transform for the last formula, we get the exact solution of the given example as:

$$
u(t, x)=\left(\frac{1-\alpha}{B(\alpha)}+\frac{2 \alpha}{\Gamma(\alpha+3) B(\alpha)} t^{\alpha}\right) t^{2} \sin x .
$$

In the next section, we construct difference scheme for the Implicit finite difference method. Then, we shall prove the stability estimates for this difference scheme method. 


\section{Stability estimates for Implicit Finite Differ- ence Method}

Suppose that $h=\frac{x_{R}-x_{L}}{M}$ for $x$-axis and $\tau=\frac{T}{N}$ for $t$-axis as grid mess, then we can write

$$
x_{n}=x_{L}+n h ; n=1,2, \ldots M, t_{k}=k \tau, k=1,2, \ldots, N .
$$

For the fractional order telegraph partial differential equation defined by $\mathrm{AB}$ derivative of the formula (1), we construct the impicit finite difference method. For this, we need to give the following formulas:

$$
\begin{aligned}
& u_{x x}\left(t_{k}, x_{n}\right) \cong \frac{u_{n+1}^{k}-2 u_{n}^{k}+u_{n-1}^{k}}{h^{2}}, \\
& u_{t t}\left(t_{k}, x_{n}\right) \cong \frac{u_{n}^{k+1}-2 u_{n}^{k}+u_{n}^{k-1}}{\tau^{2}} .
\end{aligned}
$$

And from the article [26], we know that

$$
{ }_{0}^{A B C} D_{t}^{\alpha} u\left(t_{k}, x_{n}\right) \cong \frac{1}{\Gamma(\alpha)} \sum_{j=0}^{k} \frac{u_{n}^{k+1}-u_{n}^{k}}{\tau} d_{j, k},
$$

where $d_{j, k}=\left(t_{j}-t_{k+1}\right)^{1-\alpha}-\left(t_{j}-t_{k}\right)^{1-\alpha}$. From the last three formula, we can obtain difference schemes for the fractional order telegraph equation

$$
\left\{\begin{array}{l}
\frac{u_{n}^{k+1}-2 u_{n}^{k}+u_{n}^{k-1}}{\tau^{2}}+\frac{1}{\Gamma(\alpha)} \sum_{j=0}^{k} \frac{u_{n}^{k+1}-u_{n}^{k}}{\tau} d_{j, k}-\frac{u_{n+1}^{k}-2 u_{n}^{k}+u_{n-1}^{k}}{h^{2}}=f_{n}^{k}, \\
f_{n}^{k}=f\left(t_{k}, x_{n}\right), \\
u_{n}^{0}=h_{1}\left(x_{n}\right), \frac{u_{n}^{1}-u_{n}^{0}}{\tau}=h_{2}(x), 0 \leq n \leq M, \\
u_{0}^{k}=u_{M}^{k}=0,0 \leq k \leq N .
\end{array}\right.
$$

The last difference formula can be rewritten in the following form

$$
\left\{\begin{array}{l}
\left(\frac{1}{\tau^{2}}+\frac{d_{j, k}}{\tau \Gamma(\alpha)}\right) u_{n}^{k+1}+\left(-\frac{1}{h^{2}}\right) u_{n-1}^{k}+\left(-\frac{2}{\tau^{2}}+\frac{2}{h^{2}}-\frac{d_{j, k}}{\tau \Gamma(\alpha)}\right) u_{n}^{k} \\
+\left(-\frac{1}{h^{2}}\right) u_{n+1}^{k}+\left(\frac{1}{\tau^{2}}\right) u_{n}^{k-1}=f_{n}^{k}=f\left(t_{k}, x_{n}\right), \\
u_{n}^{0}=h_{1}\left(x_{n}\right), \frac{u_{n}^{1}-u_{n}^{0}}{\tau}=h_{2}(x), 1 \leq n \leq M, \\
u_{0}^{k}=u_{M}^{k}=0,0 \leq k \leq N .
\end{array}\right.
$$

Using the initial conditions, the difference scheme formula (16) can be written in the matrix form as follows: 


$$
\left\{\begin{array}{l}
u^{1}=u^{0}+\tau h_{2}, \\
A U^{k+1}=B U^{k}+C U^{k-1}+\varphi_{n}^{k}, \\
f_{n}^{k}=f\left(t_{k}, x_{n}\right), \\
u_{n}^{0}=h_{1}\left(x_{n}\right), \frac{u_{n}^{1}-u_{n}^{0}}{\tau}=h_{2}\left(x_{n}\right), 1 \leq n \leq M, \\
u_{0}^{k}=u_{M}^{k}=0,0 \leq k \leq N,
\end{array}\right.
$$

where $\varphi_{n}^{k}=\left[\varphi_{0}^{k}, \varphi_{1}^{k}, \ldots, \varphi_{M}^{k}\right]^{T}, \varphi_{n}^{0}=h_{1}\left(x_{n}\right), \varphi_{n}^{k}=f_{n}^{k}=f\left(t_{k}, x_{n}\right), 1 \leq n \leq$ $M, 1 \leq k \leq N$, and $u^{k}=\left[u_{0}^{k}, u_{1}^{k}, \ldots, u_{M}^{k}\right]^{T}$. Here, $A$ and $C$ are symmetric tridiagonal matrixs and $B$ is diagonal matrix.

We take to $\|A\|=\|A\|_{\infty}=\max _{1 \leq k \leq N-1}\left\{\sum_{j=1}^{N-1}\left|a_{j k}\right|\right\}$, where $A=\left[a_{j k}\right]_{(N-1) \times(N-1)}$,

$I$ is unit matrix. $d_{j, k}=\max \left(\operatorname{Re}\left\{(-\tau)^{1-\alpha},(-2 \tau)^{1-\alpha}, \ldots,(-k \tau)^{1-\alpha}\right\}\right) \leq \tau$.

Lemma 6 If $-\frac{2}{\tau^{2}}+\frac{2}{h^{2}}-\frac{d_{j, k}}{\tau \Gamma(\alpha)}>0$, then $\left\|A^{-1} B\right\| \leq 1$.

\section{Proof.}

$$
\begin{aligned}
\left\|A^{-1} B\right\| \leq & \left\|A^{-1}\right\|\|B\| \leq \frac{1}{\min _{1 \leq k \leq N-1}\left\{\left|a_{k k}\right|-\sum_{\substack{m \neq k, m=1}}^{N-1}\left|a_{k m}\right|\right\}}\|B\| \\
& \leq \frac{\left|-\frac{2}{\tau^{2}}+\frac{2}{h^{2}}-\frac{d_{j, k}}{\tau \Gamma(\alpha)}\right|+\left|-\frac{1}{h^{2}}\right|+\left|-\frac{1}{h^{2}}\right|}{\left|\frac{1}{\tau^{2}}+\frac{d_{j, k}}{\tau \Gamma(\alpha)}\right|} \\
& =\frac{-\frac{2}{\tau^{2}}+\frac{4}{h^{2}}-\frac{d_{j, k}}{\tau \Gamma(\alpha)}}{\frac{1}{\tau^{2}}+\frac{d_{j, k}}{\tau \Gamma(\alpha)}} \leq 1
\end{aligned}
$$

Lemma 7 If $-\frac{2}{\tau^{2}}+\frac{2}{h^{2}}-\frac{d_{j, k}}{\tau \Gamma(\alpha)}>0$, then $\left\|A^{-1} C\right\| \leq 1$.

Proof. $\left.\left\|A^{-1} C\right\| \leq\left\|A^{-1}\right\|\|C\| \leq \frac{1}{\min _{1 \leq k \leq N-1}\left\{\left|a_{k k}\right|-\sum_{\substack{m \neq k, m=1}}^{N-1}\left|a_{k m}\right|\right.}\right\}\|C\| \leq \frac{\left|\frac{1}{\tau^{2}}\right|}{\left|\frac{1}{\tau^{2}}+\frac{d_{j, k}}{\tau \Gamma(\alpha)}\right|}=$ $\frac{\frac{1}{\tau^{2}}}{\frac{1}{\tau^{2}}+\frac{d_{j, k}}{\tau \Gamma(\alpha)}} \leq 1$

Theorem 8 If $-\frac{2}{\tau^{2}}+\frac{2}{h^{2}}-\frac{d_{j, k}}{\tau \Gamma(\alpha)}>0$. Then, the equation (17) is stable. 
Proof. Using Lemma 6 and Lemma 7, the proof of theorem is completed.

Remark 9 Convergences of implicit finite difference method was given in detail for fractional order telegraph partial differential equation in the sense of Caputo derivative in [12].

\section{Numerical implementation}

In this section, we obtain approxiation solution by using the implicit finite difference method for the Example:1 4. To solve problem (4, we shall apply the implict finite method given by the formula (15). We use a procedure of the modified Gauss elimination method for difference equation 4 . We calculate the maximum norm of the error of numerical solution as

$$
\varepsilon=\max _{\substack{n=0,1, \ldots, M \\ k=0,1,2 \ldots, N}}\left|u(t, x)-U\left(t_{k}, x_{n}\right)\right|,
$$

where $u_{n}^{k}=u\left(t_{k}, x_{n}\right)$ is the approximate solution and $u(t, x)$ is exact solution.

Example 10 Consider the fractional order telegraph partial differential equation given in Example 4. We know that the exact solution of this equation is

$$
u(t, x)=\left(\frac{1-\alpha}{B(\alpha)}+\frac{2 \alpha}{\Gamma(\alpha+3) B(\alpha)} t^{\alpha}\right) t^{2} \sin x .
$$

Error analysis table (cf. Table I) gives our error analysis for the implicit finite difference method.

The Implicit finite difference method for 4 by interval $\tau=\frac{1}{N}, h=\frac{p i}{M}$

\begin{tabular}{|l|l|l|l|l|l|}
\hline$\alpha$ & $N=M=10$ & $N=M=20$ & $N=M=40$ & $N=M=80$ & $N=M=160$ \\
\hline 0.001 & 0.0678 & 0.0727 & 0.0763 & 0.0785 & 0.0797 \\
\cline { 2 - 6 } 0.01 & 0.0711 & 0.0758 & 0.0794 & 0.0816 & 0.0827 \\
\cline { 2 - 6 } 0.05 & 0.0877 & 0.0914 & 0.0945 & 0.0964 & 0.0974 \\
\hline 0.1 & 0.1126 & 0.1140 & 0.1158 & 0.1169 & 0.1175 \\
\hline 0.25 & 0.1990 & 0.1871 & 0.1811 & 0.1778 & 0.1759 \\
\cline { 2 - 6 } 0.5 & 0.2450 & 0.2185 & 0.2044 & 0.1970 & 0.1931 \\
\cline { 2 - 6 } 0.75 & 0.1211 & 0.1058 & 0.1012 & 0.1002 & 0.1000 \\
\cline { 2 - 6 } 0.9 & 0.0636 & 0.0366 & 0.0310 & 0.0327 & 0.0348 \\
\cline { 2 - 6 } 0.95 & 0.0672 & 0.0315 & 0.0156 & 0.0132 & 0.0150 \\
\cline { 2 - 6 } 0.99 & 0.0775 & 0.0392 & 0.0187 & 0.0083 & 0.0034 \\
\hline 0.999 & 0.0804 & 0.0418 & 0.0212 & 0.0105 & 0.0052 \\
\hline 1 & 0.0807 & 0.0421 & 0.0214 & 0.0108 & 0.0054 \\
\hline
\end{tabular}

Tablo I. Error analysis is computed for the variable values $0<\alpha<1$, $0<t<1$, and $0<x<\pi$ of the approximation solution by helping the Implicit difference formula (15). 


\section{Conclusion}

In this paper, the exact solution and stability estimates are showed for the fractional order telegraph partial differential equation of the abstract form. One example of the this differential is solved by Laplace transform method. First order Implicit finite difference schemes for the equation 1 are constructed. Stability inequalities are proved for given difference schemes by using matrix stability method. We used implicit finite difference method with Atangana-Baleanu Caputo fractional definition to conctruct and obtain to accuracy algorithms for solving telegraph partial differential equations. Approximate solutions for numerical experiments are found by the implicit finite difference-method. These results were compared with the exact solutions. MATLAB program is used for all numerical calculations. From the Figure 1. and Figure 2., physical properties of the exact and the approximate solutions can be seen for the interval $N=M=10$. In Figure 1. and Figure 2., the exact and approximation solutions are shown for $\alpha=\frac{1}{2}, \operatorname{tau}=\frac{1}{10}$ and $h=\frac{\pi}{10}$, respectively. The exact solution is obtained as 1.024046087059265 and the approximation solution is obtained as 1.149063312280427. We note that the absolute error is equal to value given in Table I. for $\alpha=\frac{1}{2}, \operatorname{tau}=\frac{1}{10}$ and $h=\frac{\pi}{10}$. From the mathematical and physical point of views, it can be observed that the numerical results and Figures show the expected physical properties. To the best of our knowledge, investigation of such properties of model considered in this paper has not been submitted in advance. This method can be applied for various nonlinear fractional order partial differential equation in the future researchs.

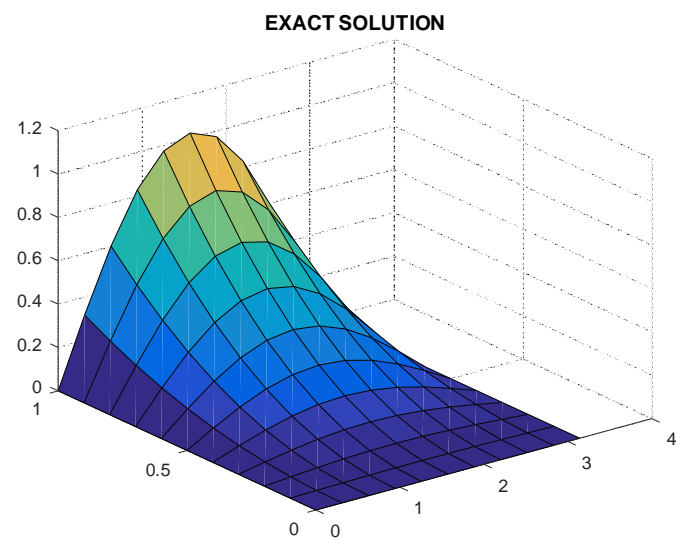

Figure 1.

Figure 1. is computed for example 10 for $\alpha=\frac{1}{2}$, tau $=\frac{1}{10}$ and $h=\frac{\pi}{10}$. 


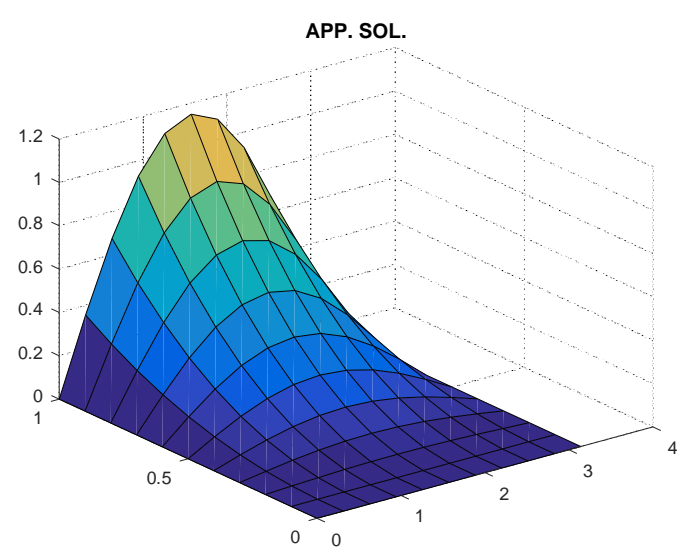

Figure 2.

Figure 2. is computed for example 10 for $\alpha=\frac{1}{2}, \operatorname{tau}=\frac{1}{10}$ and $h=\frac{\pi}{10}$. 


\section{References}

[1] Bulut, H., Kumar, D., Singh, J., Swroop, R., and Baskonus, H.M., "Analytic study for a fractional model of HIV infection of CD4+TCD4+T lymphocyte cells," Mathematics in Natural Science 2, 33-43 (2018).

[2] Baskonus, H. M., Mekkaoui, T., Hammouch, Z., and Bulut, H., "Active Control of a Chaotic Fractional Order Economic System", Entropy 17, 5771-5783 (2015).

[3] Gencoglu, M.T., Baskonus, H.M.,and Bulut, H., "Numerical simulations to the nonlinear model of interpersonal Relationships with time fractional derivative," AIP Conference Proceedings 1798, 1-9 (2017).

[4] Baskonus, H.M., and Bulut, H., "Regarding on the prototype solutions for the nonlinear fractional-order biological population model," AIP Conference Proceedings 1738, 290004 (2016).

[5] Mohyud-Din, S.T., "Homotopy analysis method for solving the space and time fractional KdV equations," Int. J. Numer. Meth. Heat Fluid Flow 22, 928-941 (2012).

[6] Gepreel, K.A., "The homotopy perturbation method applied to the nonlinear fractional Kolmogorov-Petrovskii-Piskunov equations," Appl. Math. Lett. 24, 1428-1434, (2011).

[7] Bin, Z. "(G'/G)-expansion method for solving fractional partial differential equations in the theory of mathematical physics," Communications in Theoretical Physics 58(5), 623

[8] Çelik, C., and Duman, M., "Crank-Nicolson method for the fractional diffusion equation with the Riesz fractional derivative," Journal of computational physics 231(4), 1743-1750 (2012).

[9] Gorial, I. I., "Numerical methods for fractional reaction-dispersion equation with Riesz space fractional derivative," Engineering and Technology Journal 29(4), 709-715 (2011).

[10] Jafari, H., and Daftardar-Gejji, V., "Solving linear and nonlinear fractional diffusion and wave equations by Adomian decomposition," Applied Mathematics and Computation 180(2), 488-497 (2006).

[11] Ashyralyev, A., and Modanli, M., "An operator method for telegraph partial differential and difference equations," Boundary Value Problems 2015(1), 41 (2015).

[12] Modanl, M., "Two numerical methods for fractional partial differential equation with nonlocal boundary value problem," Advances in Difference Equations 2018(1), 333 (2018). 
[13] Modanli, M., and Akgül, A., "Numerical solution of fractional telegraph differential equations by theta-method," The European Physical Journal Special Topics, 226(16-18), 3693-3703 (2018).

[14] Atangana, A., AND Koca, I., "Chaos in a simple nonlinear system with Atangana-Baleanu derivatives with fractional order," Chaos, Solitons \& Fractals, 89, 447-454 (2016).

[15] Alkahtani, R.T., "Chua's circuit model with Atangana-Baleanu derivative with fractional order," Chaos, Solitons \& Fractals, 89, 547-551 (2016).

[16] Owolabi, K.M., and Atangana, A., "Chaotic behaviour in system of noninteger-order ordinary differential equations," Chaos, Solitons \& Fractals, 115, 362-370 (2018).

[17] Atangana, A., and Baleanu, D., "New fractional derivatives with nonlocal and non-singular kernel: theory and application to heat transfer model," arXiv preprint arXiv:1602.03408 (2016).

[18] Gomez-Aguilar, J. F., Yepez-Martinez, H., Escobar-Jiménez, R. F., Astorga-Zaragoza, C. M., and Reyes-Reyes, J., "Analytical and numerical solutions of electrical circuits described by fractional derivatives," Applied Mathematical Modelling, 40(21-22), 9079-9094 (2016).

[19] Ghanbari, B., and Gómez-Aguilar, J. F., "Modeling the dynamics of nutrient-phytoplankton-zooplankton system with variable-order fractional derivatives," Chaos, Solitons \& Fractals, 116, 114-120 (2018).

[20] Saad, K. M., and Al-Sharif, E. H., "Analytical study for time and timespace fractional Burgers' equation," Advances in Difference Equations, 2017(1), 300 (2017).

[21] Saad, K. M., Atangana, A., and Baleanu, D., "New fractional derivatives with non-singular kernel applied to the Burgers equation," Chaos: An Interdisciplinary Journal of Nonlinear Science, 28(6), 063109 (2018).

[22] Abbott, M. B., and Basco, D. R., "Computational fluid dynamics-an introduction for engineers," NASA STI/Recon Technical Report A, 90 (1989).

[23] Yépez-Martínez, H., Gómez-Aguilar, J. F., Sosa, I. O., Reyes, J. M., and Torres-Jiménez, J., "The Feng's first integral method applied to the nonlinear $\mathrm{mKdV}$ space-time fractional partial differential equation," Revista Mexicana de Física, 62(4) (2016).

[24] Coronel-Escamilla, A., Gómez-Aguilar, J. F., Baleanu, D., Córdova-Fraga, T., Escobar-Jiménez, R. F., Olivares-Peregrino, V. H., and Qurashi, M. M. A., Bateman-Feshbach Tikochinsky and Caldirola-Kanai Oscillators with New Fractional Differentiation. Entropy, 19(2), 55 (2017). 
[25] Atangana, A.,and Baleanu, D., "New fractional derivatives with nonlocal and non-singular kernel: Theory and application to heat transfer model," Thermal Science, 20,763-769 (2016).

[26] Alqahtani,R.T., "Atangana-Baleanu derivative with fractional order applied to the model of groundwater within an unconfined aquifer," J. Nonlinear Sci. Appl, 9, 3647-3654 (2016). 